\title{
Proceedings of the Association of British Neurologists from the Joint Meeting with the Deutsche Gesellschaft für Neurologie, Freie Universität Berlin, 16-17 June 1995
}

HAEMATOLOGICAL ABNORMALITIES IN CEREBRAL VENOUS THROMBOSIS

RW Ross Russell. St Thomas' Hospital, London, UK

A review of 40 personally studied cases and a survey of the literature shows that about $50 \%$ of patients with sinovenous thrombosis have a demonstrable haematological abnormality. This may be a genetically determined prothrombotic state such as protein $C$ or antithrombin 111 deficiency, an acquired thrombotic state such as antiphospholipid syndrome, a myeloproliferative disorder such as polycythaemia or thrombocythaemia, or various forms of anaemia such as sickle cell disease and haemolytic anaemia. Cerebral venous thrombosis may also be a non-metastatic complication of malignant disease such as pancreatic carcinoma or of a systemic disease such as nephrotic syndrome or ulcerative colitis. In inherited prothrombotic disorders there are usually other provoking features such as pregnancy, postoperative state, and oral contraceptive medication

The clinical features are extremely variable and consist of a combination of intracranial hypertension, seizures, foca infarction (often haemorrhagic) and cerebral oedema. Onset can be acute, subacute, or chronic and many different sites may be involved including the cerebellum and basal ganglia.

Long term anticoagulant treatment is effective and is not as dangerous as was once thought. Many patients make a full recovery.

\section{ARTERIOSCLEROTIC PARKINSONISM}

Angus P Nisbet, Susan E Daniel, Andrew J Lees. Parkinson's Disease Society Brain Bank, London, UK

The clinicopathological features of nine cases with a parkinsonism, considered to be caused by cerebrovascular disease, are described. The mean age of onset of symptoms was 68.8 years with a mean duration of disease to death of $8 \cdot 2$ years. Seven had been diagnosed clinically as Parkinson's disease and common classic features included facial freezing, a shuffling reduced steppage gait, limb and axial lead pipe rigidity and cogwheeling, difficulty rising from chair, slurred low volume speech, absent arm swing, micrographia, drooling, tremor, and diurnal variation in symptoms. Atypical features included an abrupt onset and early balance disturbance with falls, urinary incontinence, and pronounced cognitive impairment, pyramidal signs, and the presence of cerebrovascular risk factors.

All nine had fibrohyalinosis of arterioles and small arteries with extensive ischaemic damage to the striatum and hemispheric white matter with associated dilatation of the lateral ventricles. The substantia nigra was largely uninvolved and significant large vessel cerebrovascular disease was only present in two cases. The existence of a parkinsonism due to cerebrovascular disease is supported and the pathology in all cases is small vessel cerebrovascular disease indistinguishable from Binswanger's encephalopathy

BOTULINUM TOXIN TREATMENT IN STROKE PATIENTS WITH SEVERE UPPER LIMB SPASTICITY

B Bhakta, JA Cozens, JM Bamford, MA Chamberlain. Rheumatology and Rehabilitation Research Unit, University of Leeds, UK

Severe spasticity can be a contributing factor in patients with poor recovery of upper limb function after stroke. Botulinum toxin offers local treatment of spasticity without affecting sensation.

Fifteen patients $(5 \mathrm{M}, 10 \mathrm{~F})$ with severe spasticity and a non-functioning arm were treated with botulinum toxin (age range 44-79, mean 53.2 years; mean time from stroke 5.9 years). Problems defined by patients were difficulty with cleaning the palm (15/15); cutting fingernails (15/15) putting the arm through a sleeve (14/15) standing and walking balance (3/15); and pain in the shoulder $(7 / 15)$, elbow $(2 / 15)$ and wrist (6/15). Outcome measures used were (1) passive goniometry at shoulder elbow, wrist and, fingers; (2) Ashworth scale; (3) an eight point patient completed scale to assess functional change for each problem defined by the patient (successful outcome defined as a two point change in this score); and (4) pain score.

Elbow and forearm finger flexor muscles were injected; total dose per patient: 400-1000 mouse units (MU) of Dysport (Speywood); 100-200 MU of BOTOX (Allergan).

Hand hygiene improved in $12 / 15(80 \%)$; difficulty with sleeves improved in $3 / 14$ $(21 \%)$; standing and walking balance improved in 1/3; shoulder pain improved in 4/7 (57\%); wrist pain improved in $5 / 6$ $(83 \%)$. Passive movement at wrist (change $>20^{\circ}$ ) improved in $11 / 15(73 \%)$. Func- tional improvement occurred without change in the Ashworth scale. Benefit was observed within two weeks and lasted one to eight months. No adverse effects occurred.

USE OF MRI AND MRS IN THE PREDICTION OF CLINICAL OUTCOME IN PATIENTS WITH MCA INFARCTION

DE Saunders, FA Howe, A van den Boogart, JR Griffiths, MM Brown. St George's Hospital Medical School, London, UK

The purpose was to determine whether the initial metabolite concentration within the centre of the infarct, determined by proton magnetic resonance spectroscopy ( ${ }^{1} \mathrm{H}-$ MRS), and the infarct volume, determined by MRI, would be useful predictors of clinical outcome in patients with middle cerebral artery (MCA) territory infarcts.

Diagnostic MRI and short echo time ' $\mathrm{H}$ MRS were performed on a $1.5 \mathrm{~T}$ system (Signa, GE). Localised ' $\mathrm{H}-\mathrm{MRS}$ was carried out within the area of infarction and contralateral hemisphere. Peak area estimates were obtained using the variable projection time domain fitting analysis with water as an internal standard. Infarct volume was measured using the volume estimator algorithm of the ANALYZE software. Twenty patients with acute MCA infarcts were imaged within 72 hours of stroke onset; 11 had MRS performed. Clinical outcome was determined at 3 months by dividing patients into three groups; independent, dependent, and died.

One way analysis of variance (ANOVA) showed a significant difference in the initial stroke volume $(F=4.09, P<0.05)$ in the three outcome groups and demonstrated the larger the initial stroke volume the worse the clinical outcome. Changes were seen in the initial infarct spectra compared with the contralateral spectra but there was no significant difference in the metabolite concentrations in the three outcome groups.

In conclusion, MCA infarct volume determined by $\mathrm{MRI}$ in patients studied within 72 hours of stroke onset may be a valuable tool in predicting stroke outcome. Metabolite concentrations determined by ${ }^{1} \mathbf{H}-M R S$ are unhelpful in predicting stroke outcome.

EVOLUTION OF COGNITIVE IMPAIRMENT IN PARKINSON'S DISEASE

C Collinson, HJ Sagar, RA Grünewald, SM 
Doherty, J Cooper. Department of Clinical Neurology, University of Sheffield, UK

Cognitive impairment in idiopathic Parkinson's disease (IPD) may be related to neurochemical or intrinsic cortical pathology. There are no well established clinical predictors of rapid cognitive decline. One hundred and fifty untreated patients with IPD underwent evaluation of motor function, cognition, and affect at diagnosis, after randomisation to treatment, and serially over five years thereafter.

Newly diagnosed untreated patients showed impairments in memory (for example, logical memory, $P=0.01$ ) and executive function (picture arrangement (McFie), $P=0.005$ ) which correlated poorly with severity of motor disability and depression. Re-evaluation after treatment showed that dopaminergic stimulation partially improved working memory capacity (for example, digit ordering, $\mathrm{P}=$ 0.05 ) whereas anticholinergic treatment produced memory registration deficits (BrownPeterson distraction test, $P=0.01$ ). In general, cognitive decline correlated with age at diagnosis. A subgroup of patients who demented showed at diagnosis greater age, cognitive, and motor disability, yet a shorter duration of symptoms.

Cognitive impairment is detectable at diagnosis in IPD, and its rate of progression varies. A subgroup with worse prognosis for cognitive outcome can be differentiated at diagnosis Dopaminergic and cholinergic systems affect specific aspects of cognition, but progressive cognitive decline in IPD is largely due to age related non-dopaminergic neurodegeneration.

IMMUNOHISTOCHEMISTRY PROVIDES A NEUROPATHOLOGICAL CLASSIFICATION OF THE CAUSES OF FRONTOTEMPORAL DEMENTIA

PN Cooper, D Neary, DMA Mann. University of Manchester, Manchester, UK

Frontotemporal dementia (FTD) is a clinical syndrome with several pathological substrates, including frontal lobe degeneration (FLD), Pick's disease (PD), motor neuron disease dementia (MND-D), corticobasal degeneration (CBD), and cases of atypical Alzheimer's disease (AD). Clinical and pathological distinctions are not clear and overlaps have been suggested between CBD and PD, MND-D and FLD, and FLD and PD. Patients with a primary progressive language disorder are clinically described as progressive aphasia (PA).

Twenty eight brains were examined, with immunohistochemistry, from patients with clinical FTD; including cases of FLD (11 brains), PD (three), MND-D (five), PA (five), CBD (one) and "frontal" $\mathrm{AD}$ (three). CBD was identified by tau + ve inclusions, and $\beta$-amyloid and tau immunostaining defined unusual cases of $A D$. Ubiquitinated inclusions were seen in MND-D. $\alpha \mathrm{B}$ crystallin immunostaining identified cases with many cortical swollen neurons; including all PD and two cases of FLD with marked GFAP immunoreactive gliosis. These five cases also showed, by antiferritin immunostaining a cortical microglial reaction, not otherwise seen in FLD; $\alpha \mathrm{B}$ crystallin and ferritin immunostaining may help better define PD. PA and MND-D lacked cortical microglia and swollen neurons.

A protocol for the structured histopathological investigation of brains from patients with frontal dementia can therefore be derived.
BEHAVIOUR DISTURBANCES WITH

VIGABATRIN, A RETROSPECTIVE STUDY

MR Trimble, L Thomas, B Schmitz, HA Ring. Institute of Neurology, Queen Square, London, UK and Abteilung fur Psychiatrie Rudolf-Virkov Klinikum, Eschenallee 3, 14050 Berlin, Germany

The case records of four groups of patients have been examined retrospectively. The first had psychosis as a treatment emergent effect of vigabatrin, the second were patient with epilepsy and psychosis who had neve received vigabatrin, the third had depression as a treatment emergent effect of vigabatrin and the fourth were patients treated with vigabatrin who had no behavioural complications.

The variables were examined that may be associated with the development of a behavioural syndrome, with the prescription of vigabatrin. Specifically, patients developing a psychosis showed a pronounced incidence of seizure freedom, after prescription of vigabatrin. Further, patients tended to be prescribed higher doses of vigabatrin than those who did not develop psychiatric problems, and had more severe epilepsy with an earlier age of onset of epilepsy, more bilateral EEG abnormalities, and more tonic clonic seizures.

The psychiatric history showed that none of the patients who developed an affective disorder had a history of psychosis, $50 \%$ had a history of depression. In the psychosis group, nearly $30 \%$ had a history of psychosis.

FLUCTUATING NEUROPSYCHOLOGY IN CORTICAL LEWY BODY DISEASE

M Doran, N Priestley, E Ghadiali, K Wilson. The Walton Centre, Liverpool, UK

The Lewy body is the defining neuropathological feature of both Parkinson's and cortical Lewy body disease (CLBD). Some authors have considered that the two disease form opposite ends of a continuum of a common pathological process. The reported neuropsychological features of CLBD include cognitive fluctuation, attentional difficulties, and impaired visuospacial functioning.

In this study the neuropsychological profiles were compared of patients with mild (eight patients Hoehn and Yahr 1) and severe (eight Hoehn \& Yahr 3) Parkinsons disease as well as CLBD (eight patients); diagnosed according to standard criteria. A short battery of tests has been developed to quantitate the degree of cognitive fluctuation which could be administered by the patient's spouse.

The cognitive profiles of the patients with PD and CLBD were different with the patients with CLBD being better at certain non-motor tasks than either group of PD patients. The cognitive fluctuation in CLBD was not present in either PD group and showed more than $2 \mathrm{SD}$ variation for all CLBD patients over the period of one week.

The study does not lend support to the suggestion that increasing cortical Lewy body load in PD leads to a clinical state that resembles CLBD.

PATTERNS OF INHERITANCE IN UNITED KINGDOM FAMILIES WITH AMYOTROPHIC I.ATERAI. SCLEROSIS
RW Orrell, AW King, RJM Lane, J de Belleroche. Charing Cross and Westminster Medical School, London, UK

Around $5 \%-10 \%$ of patients with amyotrophic lateral sclerosis (ALS) have at least one other affected family member with the condition. Pedigrees of 105 United Kingdom families were ascertained with ALS. These include one conjugal instance of ALS which is not considered further in this analysis. Gene mutations or reduced enzyme activity of copper-zinc superoxide dismutase (SOD-1) have been identified in 13/77 $(17 \%)$ of the families in whom DNA was available from at least one affected member. $24 / 105(23 \%)$ of families contain only two affected patients in the same generation, in whom the cause might be attributed to an autosomal recessive gene, or environmental causes. However, two of these families have evidence of an SOD-1 gene mutation. These mutations are inherited in an autosomal dominant pattern as exhibited in other families. Examination of three, four, and five generation families emphasises the incomplete and variable penetrance of the SOD-1 gene mutations. These findings are important in advising patients and families on the possibility of inheriting ALS. Further analysis of the non-SOD-1 pedigrees does not support the recent suggestion of a gene with a dynamic mutation leading to selective influence of ancestral sex on transmission of ALS.

THE POSTIRRADIATION LOWER MOTOR NEURON SYNDROME: NEURONOPATHY OR RADICULOPATHY?

J Bowen, R Gregory, W Squier, $M$ Donaghy. University of Oxford and Radcliffe Infirmary, Oxford, UK

It is not known whether the postirradiation lower motor neuron syndrome results from radiation damage to motorneuron perikarya, or from damage to the nerve roots of the cauda equina. Six cases were studied who had presented with testicular neoplasms, subsequently undergoing irradiation that encompassed inter alia paraaortic nodes with coirradiation of the distal spinal cord and cauda equina. A predominantly motor disorder affecting the legs ensued after variable and often prolonged latencies (3-25 years). However, all patients also developed mild sensory features either initially or on prolonged follow up. Sural sensory nerve action potentials were normal in five. Mild sphincter symptoms occurred in three of five surviving cases after a mean of 7.9 years Magnetic resonance imaging showed gadolinium enhancement of the cauda equina in two of three patients. The first reported neuropathological study of the conus and cauda equina was performed in one of our patients who died with this condition. This showed a radiation induced vasculopathy of the proximal spinal roots, with preservation of anterior horn cells and spinal cord architecture. These clinical, radiological, neurophysiological, and pathological findings all point to a predominantly, bu not exclusively, motor radiculopathy affecting roots within the irradiated portion of the cauda equina proximal to the dorsal root ganglia. There is no evidence that radiation induced motor neuron loss from the distal spinal cord is responsible. 
FREE RADICAL DEFENCE MECHANISMS IN SPORADIC MOTOR NEURON DISEASE

JD Mitchell, PS Fitzmaurice, K Rajamani, IC Shan. Department of Neurology, Roya Preston Hospital, Preston and Centre for Toxicology, University of Central Lancashire, Preston, UK

Evidence of abnormal free radical activity in sporadic motor neuron disease (MND) has been sought for more than 10 years. Other workers have now found mutations of the $\mathrm{Cu} / \mathrm{Zn}$ superoxide dismutase (SOD1) gene on chromosome 21 in some kindreds with familial MND. Although reduced spinal cord activities of glutathione peroxidase (GSHPX) was previously found in patients dying of sporadic MND, GSHPX activity in anterior horn tissue has not been demonstrable down to a limit of $1.5 \times 10^{-4}$ units $/ \mathrm{mg}$. There is also evidence of a serial decline in erythrocyte GSHPX activity with disease progression $(P<0.001)$ and measured SOD isoenzyme activities in anterior horn tissue from eight patients who have died of histologically established sporadic MND and five non-neurological controls. Although total SOD activities were comparable in both groups, SOD1 activity was lower in the patients with MND with a compensatory upregulation $(P=0.0041$ in thoracic cord) of manganese (mitochondrial) SOD. There was no evidence of significant cytochrome P450 activity in the cervical cord. This is the first time that anterior horn SOD activities have been measured and these findings raise fundamental questions regarding the handling of environmental toxins and disruption of free radical defence mechanisms in sporadic MND.

SWALLOWING IN HEALTHY ADULTS AND IN PATIENTS WITH MOTOR NEURON DISEASE

TAT Hughes, CM Wiles. Department of Medicine (Neurology), University of Wales College of Medicine, Cardiff, UK

One hundred and eighty one carefully screened asymptomatic adults (90 men aged 18.9-91.3 years) and 30 patients (13 male, aged $39 \cdot 3-85.3$ years) with motor neuron disease (MND) at presentation were examined. They answered a swallowing questionnaire and performed a timed test of swallowing. In healthy men and MND patients who perceived a swallowing problem, median average volume per swallow (VS) was $25.0 \mathrm{ml}$ (interquartile range $21 \cdot 4-37 \cdot 5)$ and $7 \cdot 3 \mathrm{ml}(4 \cdot 5-13 \cdot 8)$, median average time per swallow $1 \cdot 2 \mathrm{~s}(1 \cdot 1-1 \cdot 4)$ and $2 \cdot 3 \mathrm{~s}(1 \cdot 4-3 \cdot 5)$, and mean swallowing capacity (VT) $23.8 \mathrm{ml} / \mathrm{s}$ (SD 9.7) and $3.1 \mathrm{ml} / \mathrm{s}$ (6.3) respectively; corresponding results in women were $16.7 \mathrm{ml}(13.6-21.4)$ and $4.0 \mathrm{ml}$ $(2 \cdot 0-5 \cdot 3), \quad 1.3 \mathrm{~s} \quad(1 \cdot 1-1 \cdot 7)$ and $3.2 \mathrm{~s}$ $(2.4-4.3)$, and $13.7 \mathrm{ml} / \mathrm{s}(6.0)$, and $1.6 \mathrm{ml} / \mathrm{s}$ $(1 \cdot 5)$. Age, sex, and height are predictors of $\log$ VT $\left(R^{2}\right.$ adjusted $\left.55.6 \%, P<0.0001\right)$ in healthy adults.

The timed test allows swallowing function in MND to be quantified on an interval scale and related to age and sex matched controls. Normative clinical data allow bulbar symptoms and signs to be more fully interpreted.

DIFFERENTIAL DIAGNOSIS AND DIAGNOSTIC CLINICAL FEATURES OF CREUTZFELDT-JAKOB DISEASE
RN de Silva, RG Will, TFG Esmonde, $M$ Zeidler. National CJD Surveillance Unit, Western General Hospital, Edinburgh, UK

Creutzfeldt-Jakob disease (CJD) is a rapidly progressive neurodegenerative disorder. Between May 1990 and April 1994, 40 cases of suspect CJD referred to the United Kingdom national surveillance unit were shown at necropsy to have succumbed to another neurodegenerative process. These included Alzheimer's disease (the commonest differential diagnosis, being present in 23 out of the $\mathbf{4 0}$ cases), multi-infarct disease, Lewy body disease, motor neuron disease, Pick's disease, corticobasal degeneration, progressive supranuclear palsy, multiple system atrophy and idiopathic cerebellar degeneration.

Review of the clinical features of these cases showed that seven fulfilled Masters' criteria for possible CID during life. Clinical characteristics were compared with 144 sporadic and 14 familial cases of definite or probable CID. Mean disease duration in the non-CJD group was 31 months (range 1 to 151 , median 16) whereas in the CJD group it was eight months (range 1 to 112 , median 4). The relative sensitivities and specificities of the common clinical features of CJD were as follows:

\begin{tabular}{lll}
\hline Sign & $\begin{array}{l}\text { Sensi- } \\
\text { tivity }\end{array}$ & $\begin{array}{l}\text { Specifi- } \\
\text { city }\end{array}$ \\
\hline Cognitive impairment & $\mathbf{0 . 9 8 1}$ & $\mathbf{0 . 1 4 3}$ \\
Myoclonus & $\mathbf{0 . 8 4 8}$ & $\mathbf{0 . 5 0 0}$ \\
Cortical visual disturbance & $\mathbf{0 . 6 4 6}$ & 0.725 \\
Pyramidal signs & $\mathbf{0 . 5 8 9}$ & 0.500 \\
Extrapyramidal features & $\mathbf{0 . 5 1 3}$ & 0.650 \\
Cerebellar dysfunction & $\mathbf{0 . 8 4 8}$ & 0.650 \\
Akinetic mutism & $\mathbf{0 . 7 2 2}$ & $\mathbf{0 . 8 0 0}$ \\
Muscle wasting & $\mathbf{0 . 1 6 5}$ & $\mathbf{0 . 8 5 0}$ \\
Characteristic EEC & $\mathbf{0 . 4 4 8}$ & 1.000 \\
\hline
\end{tabular}

OLFACTORY DISORDER IN MOTOR NEURON DISEASE

CH Hawkes, B Shephard, JF Geddes, GD Body, JE Martin, SE Daniel. Department of Clinical Neurology, Ipswich Hospital, Ipswich, Department of Morbid Anatomy, Royal London, Hospital Medical College, London, and Institute of Neurology, Queen Square, London, UK

Olfaction is impaired in degenerative conditions such as Parkinson's and Alzheimer's disease and it was suggested that olfactory identification is abnormal in motor neuron disease (MND).

Olfaction was assessed by (a) University of Pennsylvania Smell Identification Test (UPSIT) in 48 patients compared with 134 controls; (b) olfactory evoked response to hydrogen sulphide; (c) pathological examination of olfactory bulbs in eight patients and eight age matched controls. Patients were excluded if the mini mental examination score was below 27/30. All corresponded to the clinically definite or probable groups of MND by Escorial criteria.

(a) Overall UPSIT scores were normal (P $=0.65$, regression analysis). However, patients showed particular difficulty in identification of cheese-like odours; (b) olfactory evoked responses were normal; (c) pronounced abnormalities were seen in all olfactory bulbs with lipofuscin containing cells in $>95 \%$ cases compared with $<50 \%$ controls.

In conclusion, the clinical changes in smell function are subtle. The selective difficulty with cheese-like odour suggests specific damage to olfactory receptor cells perhaps caused by exogenous agents. Excess lipofuscin deposition is a well recognised feature of diseased motor cells in MND but its presence in olfactory neurons has not been described. Such deposits characterise lipid peroxidation and suggest that increased free radical damage may be occurring in MND as implied by the discovery of defective superoxide dismutase gene in some familial (and possibly sporadic) forms of MND.

RECURRENT RHABDOMYOLYSIS DUE TO DISORDERS OF FAT METABOLISM

J Schaefer, S Jackson, DJ Dick, DM Turnbull. Division of Clinical Neuroscience, University of Newcastle upon Tyne, UK, Department of Neurology, University of Wuerzburg, and Department of Neurology, Norfolk and Norwich Hospital, Norwich, UK

Recurrent attacks of rhabdomyolysis and myoglobinuria are an important and life threatening feature of certain muscle diseases. Probably the most important cause of myoglobinuria to recognise is a defect of mitochondrial fatty acid oxidation. Patients with these defects may present with recurrent episodes of myoglobinuria, but are also at considerable risk from hypoglycaemia and cardiomyopathy. Defects of fatty acid oxidation are not only one of the commonest inborn errors of metabolism, but are also easily treated by dietary management. Thus a potentially fatal outcome can be prevented by simply ensuring that patients have an adequate supply of carbohydrate.

In 10 patients with recurrent episodes of myoglobinuria due to defects of mitochondrial fatty acid oxidation we have shown that despite a similar presentation four different enzyme defects were found. Thus simply screening patients for CPT deficiency is insufficient in this patient group. We have developed a screening technique which allows for the rapid diagnosis of all defects of fatty acid oxidation using only blood or skin biopsy sample. Using this technique, we have identified a family who have a defect of a recently identified enzyme, the trifunctional protein (TFP). Whereas most reported patients with TFP deficiency die in early childhood, the affected members in this family presented in adult life. There was pronounced clinical heterogeneity in this family, ranging from death secondary to rhabdomyolysis and renal failure, to an asymptomatic family member in his late thirties. This particular enzyme defect is often associated with a peripheral neuropathy, which is an important clinical feature in this family.

GLIAL TRANSPLANTATION IN THE REPAIR OF DEMYELINATED LESIONS

J Sussman, MP Targett, NJ Scolding, WF Blakemore, DAS Compston. University of Cambridge Neurology Unit, Cambridge, UK

Multiple Sclerosis (MS) is a demyelinating disorder in which inflammatory damage results in the loss of oligodendrocytes and their myelin sheaths. Pathological studies in 
early MS show that extensive remyelination may take place, and suggest that repeated episodes of demyelination within individual sites result in hypocellular lesions which do not remyelinate. Progenitor cells which are capable of division and differentiation into oligodendrocytes have been described in tissue cultures derived from adult rodent and human brain, and it has been suggested that these cells might have become exhausted in chronic hypocellular MS lesions.

To investigate the capacity for remyelination by glial cells derived from the adult CNS, a demyelinated lesion was created by using ethidium bromide injection into the irradiated dorsal columns of adult rats. Glial cells from adult CNS were transplanted into these lesions. Adult rat brainstem glia generated compacted myelin with histologically normal-appearing nodes of Ranvier. Cell numbers suggest that proliferating oligodendrocyte progenitor cells, rather than mature oligodendrocytes are responsible for this repair. Oligodendrocytes derived from human and pig CNS produced processes between axons although with little myelin production, reflecting species differences. This work shows that demyelinated axons may retain the signals required to induce myelination if glia can be introduced into demyelinated lesions.

COMA DUE TO BRAIN STEM ISCHAEMIA: IS IT ALWAYS AN INDICATOR OF GRAVE PROGNOSIS?

J Bamford, C Sherrington. Department of Neurology, St James's University Hospital, Leeds, UK

Brain stem ischaemia may cause sudden impairment of consciousness with accompanying neurological signs and the prognosis is often assumed to be extremely poor. Large vessel thromboembolism is commonly reported as the cause and thrombolytic therapy has been tested.

Six patients presenting with sudden loss of consciousness and brainstem dysfunction, made a rapid recovery after an interval (up to three days) with minimal deficit. In four patients this was recurrent. Vascular risk factors were common but no embolic source was found. Magnetic resonance imaging showed infarction in two patients, transcranial Doppler and magnetic resonance angiography suggested one mild basilar stenosis but were otherwise normal. Anticoagulants or aspirin was given. Five patients had no residual deficit, no further vascular events occurred for 12 months.

It is suggested that coma due to brainstem ischaemia does not necessarily reflect extensive tissue damage and patients may make dramatic recoveries. This is perhaps due to ischaemia in a deep perforator territory and the low incidence of discovered large vessel pathology or embolic sources supports this. Intensive supportive therapy may be justified initially and further studies of thrombolysis still require angiographic confirmation of the size of vessel involved.

SUBJECTIVE POSTURAL VERTICAL IN PERIPHERAL AND CENTRAL VESTIBULAR DISORDERS

AR Bisdorff, AM Bronstein, MA Gresty, CJ Wolsley. MRC Human Movement and Balance Unit, National Hospital, London, UK
The perception of subjective postural vertical was assessed in normal subjects $(n=52)$ and patients with various peripheral vestibu$\operatorname{lar}(n=43)$ and central vestibular disorders with up or downbeat nystagmus $(n=15)$. The subjects were seated in a motorised gimbal with head and torso restrained and eyes closed. The gimbal executed 7-10 cycles of tilt around the vertical at $1.5 \%$ in either the sagittal (pitch) or coronal (roll) plane. Subjects indicated when they began to feel upright and again when they began to feel tilted with a joystick. Normal subjects felt upright within a sector of $5^{\circ}-6^{\circ}$ around vertical in both planes. The abnormalities found in some patient groups consisted of a not plane specific enlargement of the verticality sector-that is, a loss in sensitivity. Directional biases of the perception of body in space were not significant despite directional sway abnormalities, tilted subjective visual vertical, and asymmetrical vestibuloocular function in some groups. The data indicate that the perception of body vertical does not reflect asymmetries in vestibuloocular or spinal function, suggestive of independent central processing of vestibular reflexes and perception of spatial orientation.

VISUAL PSYCHOPHYSICS AND MAGNETIC RESONANCE IMAGING IN SECONDARY PROGRESSIVE MULTIPLE SCLEROSIS

PA Caruana, MB Davies, DH Foster, $R$ Williams, N Haq, CP Hawkins. Keele Multiple Sclerosis Research Group, Department of Neurology, Royal Infirmary and Communication and Neuroscience, Keele University, Stoke on Trent, UK

In established multiple sclerosis the nature of the relation between sensory deficit in the visual pathway measured psychophysically and morphological changes detected by magnetic resonance imaging is unknown. Fifty one eyes in 26 patients with secondary progressive disease were studied by visual psychophysics and MRI of the optic nerves and brain.

Psychophysical tests obtained thresholds using a PEST routine for horizontal, sinusoidally modulated gratings of $0 \cdot 25,1 \cdot 0$, and 4.0 cycles $/{ }^{\circ}$ spatial frequency drifting upwards at $0.1,0.4$, and 1.6 cycles/second respectively, with isoluminant red and green colours out of phase for preferential detection by the parvocellular system and in phase for the magnocellular system. Isoluminance at each spatial frequency was estimated by established methods. A Phillips MR scanner $(0.5 \mathrm{~T})$ was used. MRI lesion load was evaluated independently from the extent of abnormality seen on STIR images of the optic nerve (IR1400/140/25) and from areas of reduced signal in the postchiasmal pathway on $\mathrm{T} 1$ weighted images of the brain ("T1-holes"-SE 550/10) (MBD, $\mathrm{NH}$ ).

The results suggest that the parvocellular system is more affected than the magnocellular one at the two lower spatial frequencies ( $14 \%$ deficit of $9.5 \%)$. In addition, the deficit in log contrast sensitivity for both pathways is related to abnormality on MRI. With deficits more than $10 \%$, a greater proportion of MR images (both optic nerve and postchiasmal) was abnormal than with deficits less than $10 \%$ ( $t$ test, $\mathrm{P}<0.01$ ).

The parvocellular visual system seems to be involved more than the magnocellular system in secondary progressive multiple sclerosis. The deficit in both systems is related to abnormality detected by MRI in the optic nerve and postchiasmal pathway.

POSTSYNAPTIC $5 \mathrm{HT}_{1 \mathrm{~A}}$ AND $5 \mathrm{HT}_{2 \mathrm{~A}}$ RECEPTORS ARE INCREASED IN PARKINSON'S DISEASE NEOCORTEX

CPL-H Chen, JT Alder, L Bray, AE Kingsbury, PT Francis, OJF Foster. Institute of Neurology, London UK

The serotonergic (5HT) system has been shown to be affected in Parkinson's disease (PD). Lewy bodies and loss of 5HT neurons has been reported in the raphe nuclei which provide the $5 \mathrm{HT}$ innervation to the forebrain. 5HT concentrations are reduced and a decrease in the maximal binding density $\left(B_{\max }\right)$ of the 5HT reuptake site (a marker for the 5HT innervation) has been demonstrated in the striatum and neocortex. However, neocortical postsynaptic 5HT receptors have not been adequately assessed.

$B_{\max }$ and disassociation constants $(\mathrm{Kd})$ for the $5 \mathrm{HT}$ reuptake site, $5 \mathrm{HT}_{1 \mathrm{~A}}$, and $5 \mathrm{HT}_{2 \mathrm{~A}}$ receptors were measured by saturation analysis with tritiated paroxetine, $8-\mathrm{OH}$ DPAT, and ketanserin respectively, in the orbitofrontal (BA11) and temporal (BA21) cortex of eight $P D$ patients and 20 controls matched for age, sex, and storage.

Repeated measures ANCOVA with cortical $\mathrm{pH}$ and postmortem delay as covariates showed a significant increase of $8-\mathrm{OH}-$ DPAT $(F=6.04, P=0.024)$ and ketanserin $(F=9.74, P=0.006)$ but not paroxetine $\mathrm{B}_{\max }$ or the $\mathrm{Kd}$ of any ligand in patients with PD.

Increased $5 \mathrm{HT}_{1 \mathrm{~A}}$ and $5 \mathrm{HT}_{2 \mathrm{~A}}$ postsynaptic receptors may represent an upregulation in response to a $5 \mathrm{HT}$ deficit. As drugs acting on $5 \mathrm{HT}_{1 \mathrm{~A}}$ and $5 \mathrm{HT}_{2 \mathrm{~A}}$ receptors have shown antidepressant and antipsychotic effects respectively, this increase in receptor density in PD may have clinical and therapeutic implications.

A PATIENT WITH CEREBROTENDINOUS XANTHOMATOSIS, PALATAL TREMOR, AND HYPERTROPHIED OLIVES

M Chowdhury, D Wrenn. Atkinson Morley's Hospital, London, UK

Cerebrotendinous xanthomatosis is caused by a defect in sterol 27 hydroxylase, an enzyme involved in the pathway of cholesterol metabolism and bile acid synthesis. The gene locus is on chromosome two. Most cases are sporadic, but some are inherited as an autosomal recessive. It is associated with a raised serum cholestanol concentration and gives rise to childhood onset cognitive impairment, ataxia, spasticity, neuropathy, tendon xanthomata and cataracts. A few cases also have palatal tremor. A 36 year old Indian woman had all of the above features. She had an IQ (intelligence-quotient) of 56, a prominent cerebellar syndrome with ataxia, dysarthria, dysmetria, and abnormal eye movements, and pronounced lower limb spasticity. She had a left Achilles tendon xanthoma. Her electromyogram showed a demyelinating peripheral neuropathy. She had a continuous palatal tremor (documented on videotape) of 3 hertz, which cannot be voluntarily 
suppressed. Magnetic resonance imaging of her brain stem showed pronounced hypertrophy of the olives. It is thought that pathological palatal tremor is continuous, not suppressible, and is associated with hypertrophied olives. By contrast, benign essential palatal tremor tends to be intermittent, and is associated with normal sized olives.

DOES ABNORMAL EEG PREDICT EARLY DEMENTIA IN PARKINSON'S DISEASE?

C Collinson, HJ Sagar, RA Grünewald, S Doherty, J Cooper, J Gumpert, JA Jarratt. Department of Clinical Neurology, University of Sheffield, Sheffield, UK

Abnormalities of EEG are common in idiopathic Parkinson's disease (IPD), especially in demented patients, and may predict poor treatment response. The hypothesis that abnormal EEG background at diagnosis predicts early dementia in IPD was tested.

Untreated patients with IPD underwent evaluation of motor function, cognition, and affect at diagnosis.

Results in 10 patients who demented two to four years after diagnosis were compared with 20 patients matched at diagnosis for disease duration, age, age at onset of symptoms, education, and premorbid IQ but who did not dement.

A further 55 untreated patients with IPD were divided into those with minimal background slowing and those with moderate or severe background slowing on the EEG at diagnosis. Comparisons were made by Mann-Whitney $U$ test corrected for multiple comparisons.

Patients who later demented showed at diagnosis impaired executive function (digit ordering $P=0.001$ ), memory (Rey figure reproduction $\mathrm{P}=0.003$, logical memory $\mathrm{P}=$ $0 \cdot 007$ ) and motor function (KCRS score $P$ $=0.006)$, as did patients with severely abnormal EEG background at diagnosis (digit ordering $\mathbf{P}=0.003$, Rey figure reproduction $P=0.004$, logical memory $P=$ $0.07, \mathrm{KCRS}$ score $\mathrm{P}=0.005$ ).

EEG abnormalities may predict early dementia in IPD.

COMPARISON OF METHODS TO ASSESS CEREBELLAR DYSFUNCTION IN SECONDARY PROGRESSIVE MULTIPLE SCLEROSIS AND MAGNETIC RESONANCE IMAGING

MB Davies, $\mathbf{R}$ Williams, $\mathrm{N}$ Haq, CP Hawkins. Keele Multiple Sclerosis Research Group, Department of Neurology, Royal Infirmary and MRI Unit, Cornwall House, Stoke on Trent, UK

The assessment of cerebellar dysfunction in multiple sclerosis (MS) is important in evaluating progression of disability in trials of putative treatments. Thus we compared different measures of cerebellar dysfunction in patients with secondary progressive disease. Evidence suggests that areas of reduced signal on $\mathrm{T} 1$ weighted magnetic resonance imaging (MRI “T1 holes") may be more representative of tissue disruption than high signal lesions on conventional images. Measures of cerebellar dysfunction with abnormality on $\mathrm{T} 1$ weighted images were compared.

Thirty patients were evaluated clinically for cerebellar dysfunction using non-linear scales (EDSS functional systems score and ambulation index). Linear scales provided quantitative measures of upper limb cerebellar dysfunction and were compared with an equal number of age and sex matched control subjects (pegboard test, timed spiral, and timed tapping tests). A Phillips ( $0.5 \mathrm{~T})$ scanner was used. MRI lesion load in the cerebellar pathways was evaluated independently on T1 weighted images (SE 550/10, 5 mm slice thickness) (NH, MBD).

When comparing linear measures of cerebellar dysfunction, the pegboard and timed spiral tests proved more discriminatory in detecting abnormality (pegboard 88\%, timed spiral $74 \%$, and timed tapping $29 \%$ : difference between mean of patient and control group). "T1 holes" were present in the cerebellum and brainstem in 17 out of 30 patients. In general there was no significant relation between clinical disability measured by non-linear tests and the extent of abnormality on MRI. When comparing linear measures, a significant relation was found using the timed spiral test in the non-dominant hand. When the clinical deficit exceeded $50 \%$, "T1 holes" were seen in eight out of 12 patients. With lesser deficit MRI was abnormal in only three out of 16 patients $\left(\chi^{2}, \mathrm{P}<0.02\right)$

Both the pegboard and timed spiral tests proved valuable in detecting cerebellar dysfunction in patients with secondary progressive MS. A significant relation was seen between deficit in the timed spiral test and abnormality on T1 weighted MRI.

ORGANISATION OF THE RESPIRATORY CYCLE AROUND DEGLUTITION APNOEA

K Dawson, CM Wiles. University of Wales College of Medicine, Cardiff, UK

The pattern of expiration and inspiration around the time of a swallow could be important in determining the risk of aspiration in neurogenic dysphagia. Respiratory timing and duration of apnoea associated with swallow has been measured using a sensitive microanemometer to record the direction of nasal airflow and an external throat microphone to document swallow sounds. Twelve normal adults, six male and six female (age range 21-59 years) with a "dry" swallow or boluses of $5 \mathrm{ml}$ (from spoon or cup) and 20 $\mathrm{ml}$ were investigated. Deglutition apnoea was preceded and followed by expiratory airflow in $95 \%$ of swallows; the remainder were preceded by inspiration. Median swallow apnoea was $0.90 \mathrm{~s}$ (range $0.55-1.70 \mathrm{~s}$ ) for non-bolus "dry" swallows, and overall was significantly shorter in the presence of a bolus $(\mathrm{P}<$ 0.001 ), regardless of volume. Increasing age was associated with longer apnoea time. There was no difference if a bolus was taken from a spoon or a cup. These results in subjects with normal swallowing capacity differ from some previously published and provide a basis for analysis of patients with either respiratory impairment or neurogenic dysphagia.

CONTRIBUTION OF T CELL RECEPTOR GAMMA AND DELTA CHAIN GENES TO SUSCEPTIBILITY TO MULTIPLE SCLEROSIS

AG Droogan, C Kirk, SA Hawkins, S McMillan, C Graham. Department of Neurology, Royal Victoria Hospital, Belfast and Genetics Laboratory, Belfast City Hospital, UK
Genetic susceptibility to multiple sclerosis (MS) may be due to uncommon combinations of common alleles of polymorphic genes. The influence of ontogenic rearrangements of the $T$ cell receptor (TCR) repertoire on susceptibility to MS has not been investigated. Germline inheritance of TCR $\alpha \beta$ chain genes show complex and inconsistent associations with susceptibility to MS. Genes encoding the TCR $\gamma$ and $\delta$ chains are rearranged earlier and independently from those of the TCR $\alpha$ and $\beta$ chains and may contribute to the genetic profile of risk towards MS. Polymerase chain reaction and microsatellite typing methods allow multiple locus association studies to be carried out rapidly on large sample sizes. The distribution of gene-specific TCR $\mathrm{V} \delta$ and $\mathrm{V} \gamma$ (D7S528) alleles were analysed in 180 unrelated patients with clinically definite MS and 113 unrelated healthy controls. Samples were typed using fluorescently labelled primers which were visualised with "Genescan" software on an ABI 373A fluorescent fragment analyser.

No allelic association was found for either the TCR V $\gamma\left(\chi^{2} 2.69, \mathrm{P}=0.61\right)$ or $\mathrm{V} \delta\left(\chi^{2}\right.$ $8.43, P=0.30)$ microsatellites in the population of MS patients studied. Therefore TCR $\gamma$ and $\delta$ chain genes are unlikely to represent major loci for susceptibility to multiple sclerosis.

A ROLE FOR 5HT ${ }_{2 c}$ ANTAGONISTS IN THE TREATMENT OF PARKINSON'S DISEASE?

SH Fox, JM Brotchie. University of Manchester, Manchester, UK

Current treatments for Parkinson's disease (PD) are most commonly based on dopamine replacement. Unfortunately most patients develop side effects on long term therapy, including wearing off and dyskinaesiae. Novel non-dopaminergic treatments are therefore of great interest.

The neural mechanisms underlying parkinsonian symptoms are thought to involve overactivity of neurons in the output regions of the basal ganglia (substantia nigra pars reticulata, $\mathrm{SNr}$, and medial globus pallidus, GPM). $5 \mathrm{HT}$, acting via $5 \mathrm{HT}_{2 \mathrm{c}}$ receptors, has been shown to increase the cell firing in the $\mathrm{SNr}$. Additionally, $5 \mathrm{HT}_{2 \mathrm{c}}$ receptors are selectively concentrated in the $\mathrm{SNr}$ and GPM and are increased in number in the 6-hydroxydopamine(6-OHDA)-lesioned rat model of PD. We hypothesise that excessive $5 \mathrm{HT}$ stimulation may contribute to the increased activity of the SNr and GPM and hence symptoms in PD.

The current study investigated the effects of the $5 \mathrm{HT}_{2 \mathrm{c}}$ antagonist normethylclozapine and the D2 agonist quinpirole in rats in which the nigrostriatal pathway had been lesioned by unilateral injection of 6-OHDA $(2 \mu \mathrm{l} 5 \mathrm{mg} / \mathrm{ml})$. As previously described, saline treated 6-OHDA lesioned animals showed rotational behaviour characterised by slow turning towards the side of the lesion (net ipsiversive turns + 11.9 (2.9) turns $/ \mathrm{h} \quad(\mathrm{n}=8)$. Antiparkinsonian agents such as levodopa and dopamine agonists elicit contralateral rotation in this model. In otherwise untreated 6-OHDA lesioned rats, a low dose of quinpirole (intraperitoneal $0 \cdot 1$ $\mathrm{mg} / \mathrm{kg}$ ) elicited mild contraversive rotation $(-4.5 \quad(3.5)$ turns $/ \mathrm{h})$. Coadministration of quinpirole and normethylclozapine $(1 \mathrm{mg} / \mathrm{kg}$ ) dramatically increased the rotation elicited by quinpirole $(-78.8$ (33) 
turns/h, P $<0.001$ ).

These results suggest that $5 \mathrm{HT}_{2 \mathrm{c}}$ receptor blockade may have potential in the development of novel treatments for Parkinson's disease.

ANTIGLIADIN ANTIBODIES AND

NEUROLOGICAL DISEASE

M Hadjivassiliou, A Gibson, A MilfordWard, GAB Davies-Jones. Department of Neurology, Royal Hallamshire Hospital, Sheffield and Department of Immunology, Northern General Hospital, Sheffield, UK

Antigliadin antibodies are a marker of untreated coeliac disease (CD) but can also be found in subjects with a normal jejunal mucosa. Neurological dysfunction complicates $C D$ in $8 \%$ of cases.

Using an ELISA method serum Ig-A and/or Ig-G antigliadin have been estimated in 94 patients with specific neurological diagnoses (16 stroke, 12 multiple sclerosis, 10 Parkinson's disease, 56 other diagnoses), and in 49 patients with neurological dysfunction whose aetiology remained obscure despite full investigation (26 neuropathy, 21 cerebellar ataxia, five myopathy, two myelopathy). Fifty healthy blood donors formed a third group.

The proportions of subjects with positive titres for antigliadin antibodies in the three groups were 5/96, 25/49 and 6/50 respectively ( $P<0.001$ by $\chi^{2}$ analysis). Fourteen out of 21 subjects with ataxia in group 2 were positive as were 12 out of 26 with peripheral neuropathy.

If the neurological insult has an immunological basis, then the role of the antigliadin antibodies assumes importance, even in the absence of histological evidence of CD. Elimination of the antigliadin antibodies by gluten free diet could result in stabilisation or improvement of these patients' neurological dysfunction.

THE NMDA-ANTAGONIST IFENPRODIL ALIEVIATES AKINESIA IN A PRIMATE MODEL OF PARKINSONISM

NR Hughes, IJ Mitchell, JM Brotchie. Division of Neuroscience, School of Biological Sciences, University of Manchester, Manchester, UK

Recent studies have implicated excessive glutamatergic neurotransmission throughout the basal ganglia as being important in mediating the symptoms of parkinsonism. It has been suggested that the systemic administration of excitatory amino acid (EAA) antagonists could be used in the treatment of Parkinson's disease. However, widespread antagonism of EAA transmission produces side effects such as sedation. An alternative approach involving modulation of the specific EAA systems that are overactive in parkinsonism may be more beneficial. Studies have shown that ifenprodil, an antiischaemic drug, is a non-competitive antagonist at a subset of NMDA receptors which contain the NR2B subunit. NR2B-containing NMDA receptors are selectively distributed in regions of the basal ganglia associated with increased EAA transmission-namely, the neostriatum.

The bilateral 6-hydroxydopamine lesioned marmoset model has been used to examine the antiparkinsonian properties of ifenprodil. A significant increase in activity and a corresponding decrease in parkinsonian score was seen after intraperitoneal administration of ifenprodil $(2 \cdot 5-12 \cdot 5$ $\mathrm{mg} / \mathrm{kg}$ ) as compared to vehicle administration. No side effects were found at the range of doses tested. This study shows that ifenprodil may have some benefit in the treatment of Parkinson's disease.

INHIBITION OF THE LATERAL GLOBUS PALLIDUS IN PARKINSON'S DISEASE: IN SITU HYBRIDISATION EVIDENCE

O Foster, A Nisbet, A Kingsbury, CD Marsden, AJ Lees. Parkinson's Disease Society Brain Bank, Institute of Neurology, London, UK

The activity of basal ganglia GABAergic pathways was examined in postmortem brain from control subjects and patients with Parkinson's disease (PD) using in situ hybridisation for glutamate decarboxylase 67 mRNA (GAD67 mRNA). GAD67 mRNA expression was detected in all large neurons in both segments of the globus pallidus and in distinct striatal neuronal subpopulations, substantia nigra reticulata neurons and a small subpopulation of subthalamic neurons.

In $\mathrm{PD}$ there was a $50 \%$ decrease in GAD67 mRNA expression per neuron in the lateral segment of the globus pallidus (controls: mean $72 \cdot 8 u^{2}$ (SEM 8.7) of silver grain per neuron, $n=12$; PD: mean $35.9 u^{2}$ (SEM 9.7) of silver grain per neuron, $\mathbf{n}=9$, $P=0.01$, students $t$ test). In the medial segment of the globus pallidus, there was a small non-significant decrease of GAD67 mRNA expression (controls: mean $100 \cdot 6 \mathrm{u}^{2}$ (SEM 7.2) of silver grain per neuron, $n=$ 11; PD: mean 84.8 $\mathrm{u}^{2}$ (SEM 13.0) of silver grain per neuron, $\mathrm{n}=7, \mathrm{P}>0 \cdot 1$, students $t$ test). No significant differences in GAD67 mRNA were detected in striatal neuronal populations. These results are consisten with increased striatal inhibition of the lateral globus pallidus after nigral injury, as shown in animal models, and have important implications for neurosurgical intervention in PD.

CENTRAL BENZODIAZEPINE RECEPTORS IN IDIOPATHIC GENERALISED EPILEPSY AND THE EFFECT OF SODIUM VALPROATE: A

LONGITUDINAL ${ }^{11} \mathrm{C}$-FLUMAZENIL PET STUDY

MJ Koepp, MP Richardson, DJ Brooks, DR Fish, JS Duncan. Epilepsy Research Group, Institute of Neurology, London, UK and MRC Cyclotron Unit, Hammersmith Hospital, London, UK

The rational was to determine the effect of sodium valproate (VPA) on cerebral central benzodiazepine receptors (BZR) in patients with idiopathic generalised epilepsy (IGE). VPA results in increased GABA concentrations in CSF. In a cross sectional study it was shown that patients with IGE receiving VPA had a mean $8 \%$ reduction of $11 \mathrm{C}$ flumazenil volume of distribution (11CFmz-Vd).

In a longitudinal study $11 \mathrm{C}-\mathrm{Fmz}-\mathrm{Vd}$ in 10 patients with IGE aged 19-56 years was calculated using tracer alone high specific 11C-Fmz-PET. The first scan was performed when the patient was not taking VPA and the second four weeks after the addition of VPA up to the clinically indicated dose. Using statistical parametric mapping (SPM) pretreatment scans were compared with 24 age related normal volunteers.

The addition of VPA was associated with a $4 \%$ reduction of cortical $11 \mathrm{C}-\mathrm{Fmz}-\mathrm{Vd}$ SPM did not show any differences in 11Cflumazenil binding between patients with IGE and normal controls.

The result of a $4 \%$ reduction lies within the range of interscan variability of $5 \%$. Therefore VPA therapy probably has no effect on the number of GABAA-BZ receptors. VPA might act at the GABAA-BZR complex, possibly via changing the configuration but not the amount of receptors.

ASYMMETRIES OF THE HUMAN LINEAR VOR AFTER UNII ATERAL VESTIBULAR SURGERY

T Lempert, C Gianna, G Brookes, AM Bronstein, MA Gresty. MRC Human Movement and Balance Unit, Institute of Neurology, London, UK

Due to the multiple orientations of its hair cells the utricular macula has the potential to detect linear accelerations in almost any direction. To delineate the contribution of each utricular macula to the lateral linear VOR (L-VOR) patients with acute unilateral vestibular deafferentation were investigated Seven patients with Meniere's disease (six) or acoustic neuroma (one) with preserved caloric responses on the affected side underwent vestibular surgery and were investigated preoperatively (six), one week (seven), and 6 to 10 weeks after surgery (four). Testing was performed with the patient sitting upright in an electrically powered car running on a linear track. Patients were instructed to look at a target $1.5 \mathrm{~m}$ ahead before acceleration steps of $0.25 \mathrm{~g}$ along the transaural axis were administered in the dark, randomly to the left and right. The LVOR was measured by averaging the transient compensatory eye movements induced by the acceleration steps.

Before surgery the L-VOR was usually symmetric: $9 \cdot 2(4 \cdot 3) \%$ with acceleration towards the lesion (TL) and $11.7(1.8) \%$ away from the lesion (AL). Postoperatively responses became asymmetric in all patients $2 \cdot 8(3.8) \%(\mathrm{sL})$ and $13.5(4 \cdot 7) \%$ (AL) after correcting for spontaneous nystagmus. On follow up symmetry was restored with $12 \cdot 1(5 \cdot 6) \%$ s (TL) and $11.6(4.5) \%$ (AL). The sensitivity of a single otolith organ only to ipsilaterally directed acceleration steps indicates that the human transaural L-VOR originates from the lateral portion of the utricular macula.

PAROXYSMAL OCULAR TILT REACTION AND FOCAL LIMB DYSTONIA DUE TO A MESODIENCEPHALIC LESION

MA Paine, P Riordan-Eva, M Gresty, GT Plant. The National Hospital/MRC Human Movement and Balance Unit, Institute of Neurology, London, UK

Paroxysmal ocular tilt reaction consists of the triad of skew deviation, ocular torsion, and head tilt. Tilt of the subjective visual vertical is often associated. Conventionally this eye movement disorder is thought to result from abnormalities in the vestibular-otolithic pathways. A 41 year old woman with a right 
mesodiencephalic arteriovenous malformation who had features of a paroxysmal ocular tilt reaction associated with left hemidystonia is reported. She experienced frequent episodes of paroxysmal vertical diplopia. Magnetic search coil and video-oculographic recordings were performed. A paroxysm consisted of left ocular elevation with considerable conjugate clockwise binocular torsion but without significant head tilt. Associated with the paroxysm was an increase in surface EMG activity in the dystonic left arm. Between these clinically apparent paroxysms, numerous episodes of binocular torsion occurred time-locked to bursts of surface EMG activity in the left forearm flexors and extensors. The patient was started on acetazolamide with considerable subjective improvement of her ocular symptoms. Lesions of the mesodiencephalic region are recognised to cause hemidystonia and the ipsiversive paroxysmal ocular tilt reaction but this case is the first in which both have been described. The time locking indicates that the eye and limb movements are generated by the same paroxysmal neural activity.

\section{ASYMMETRY OF PROPRIOCEPTIVE}

ABNORMALITIES IN PARKINSON'S DISEASE

C Rickards, FWJ Cody. University of Manchester, Department of Neurology and Department of Biological Sciences, Manchester, UK

In healthy subjects vibration of flexor carpi radialis (FCR) tendon in the course of wrist extension movement produces undershooting of trajectory. The magnitude of this effect is significantly smaller in patients with Parkinson's disease (PD).

The effects on trajectory of vibration of FCR during the course of practised, slow $(9 \%$ ) wrist extension movements were compared between affected (A) and less affected (LA) sides in 14 patients with clearly asymmetric PD. Each patient performed a minimum of 12 vibrated (V) and 12 nonvibrated (NV) trials with each arm.

Group mean NV movement amplitudes did not differ significantly between $A$ and LA sides $(P=0.78, t$ test). Vibration produced significant reduction in extension movements on the LA (group mean V and NV amplitudes respectively, $14 \cdot 2^{\circ}$ and $19 \cdot 8^{\circ} ; \mathrm{P}<$ 0.001 , paired $t$ test) but not the A side $\left(16.9^{\circ}\right.$ and $\left.20.0^{\circ} ; \mathrm{P}=0.017\right)$. The mean ratios of V/NV were 0.68 (LA) and 0.87 (A); $\mathrm{P}=$ 0.013 , Wilcoxon. Thus the effect of vibration was diminished on the A side. For both LA and $A$ the effects of vibration were mediated through reduction in extensor EMG; the small vibration reflexes observed did not differ systematically between the sides.

These results indicate that the abnormalities of proprioceptive guidance in PD contribute to patients' motor deficits.

HETEROGENEITY IN DEGENERATIVE CORTICAL BRAIN DISEASE: THE PREDICTIVE VALUES OF DIFFERENT PATTERNS OF ABNORMALITY USING ${ }^{99}$ MC-HMPAO SPECT

PR Talbot, J Lloyd, JS Snowden, D Neary, HJ Testa. Cerebral function unit, Department of Neurology, Manchester Royal Infirmary, Manchester, UK

The purpose was to determine predictive values of different patterns of cortical blood flow
(CBF) abnormality for Alzheimer's disease (AD) and non-Alzheimer lobar atrophy (LA). ${ }_{99 \mathrm{~m}}$ Tc-HMPAO SPECT was performed on 74 patients with $A D$ and 58 patients with $L A$; comprising frontotemporal dementia (FTD), progressive aphasia (PA), and semantic dementia (SD). Regional CBF data were converted to three independent variables using principal component (PC) analysis. The first PC reflected the average CBF value, the second, anterior-posterior CBF asymmetry, and the third, left-right asymmetry. PC scores were determined for each patient and values outside $2 S D$ of the control group mean scores were considered abnormal. Predictive values were calculated using Bayes' theorem, assuming an AD:LA ratio in the general population of 10:1.

Posterior CBF asymmetry (0.99) and right sided asymmetry alone $(0.97)$ were both highly predictive of $\mathrm{AD}$, whereas anterior $\mathrm{CBF}$ asymmetry alone $(1 \cdot 00)$ was pathognomonic of FTD. Anterior asymmetry together with left sided asymmetry $(1 \cdot 00)$ occurred only in LA, although predictive values for FTD $(0.22)$, PA $(0.30)$, and SD $(0.48)$ were similar. Left sided asymmetry alone and anterior asymmetry together with right sided asymmetry were not highly predictive of either $\mathrm{AD}$ or LA.

NEMALINE MYOPATHY: AN UNUSUAL CAUSE OF OPHTHALMOPLEGIA

A Wright, GT Plant, DN Landon, JA Morgan-Hughes. The National Hospital for Neurology and Neurosurgery, London, UK

Ophthalmoplegia and ptosis are extremely rare in nemaline myopathy. A 45 year old male patient with a long history of bilateral ptosis and a one year history of diplopia is reported. Leg and arm weakness and wasting had been present since childhood with a very slow deterioration over time. On examination there was non-fatigueable bilateral ptosis which was more severe on the right. There was diplopia on left gaze. Extraocular movements showed a limitation of elevation and adduction of the right eye. There was bilateral facial weakness, as well as proximal and distal weakness and wasting in the legs and arms.

Electromyography showed a combination of myopathic and neurogenic changes. Triceps muscle biopsy showed typical changes of nemaline myopathy with small multiple collections of rod-like structures in a high proportion of fibres. The rods were usually in the central regions of the fibres but occasionally they accumulated under the membrane. Electron microscopy confirmed these findings. This patient presented with a clinical picture which did not suggest nemaline myopathy. He illustrates the heterogeneity of this disorder and the need for muscle biopsy to make an accurate diagnosis in patients with progressive ophthalmoplegia.

NEUROPSYCHOLOGICAL INVESTIGATION OF AMYOTROPHIC LATERAL SCLEROSIS (ALS)

S Abrahams, LH Goldstein, DJ Brooks, CM Lloyd, PN Leigh. Institute of Psychiatry, London, UK

In this study an extensive neuropsychological battery was employed (focusing on frontal lobe and memory functions) to inves- tigate 50 non-demented patients with typical amyotrophic lateral sclerosis (ALS), to determine whether any cognitive impairments present were exclusive to patients with pseudobulbar palsy. Two patient groups were tested (ALSpb (pseudobulbar palsy), $n=23$; ALS non-pb (non-pseudobulbar), $n=27$ ), and 31 healthy controls. The analysis of the frontal lobe tests showed a deficit on written verbal fluency, across ALS groups $(P<0.05)$, which was significantly worse in the ALSpb group $(P<$ 0.05 ). A tendency towards an impairment across groups was also found on the Wisconsin card sorting test (category, $\mathrm{P}=$ 0.06 ; errors $P=0.67$ ). Memory impairments on a word recognition test were found in both patient groups $(P<0.05)$, with a trend towards a significant impairment for picture recall $(P=0.07)$. A deficit was also found on a memory test of self ordered pointing in the ALS non-pb group $(\mathrm{P}<$ $0.05)$. These results show that patterns of frontal lobe and memory dysfunction are present across patients with ALS (consistent with our PET imaging studies). Frontal lobe deficits were more striking in patients with pseudobulbar palsy, but were not restricted to this group. Such cognitive changes seem to be an integral part of ALS.

RISK FACTOR ANALYSIS IN SPORADIC MOTOR NEURON DISEASE

JD Mitchell, A Al-Hamad, AC Gatrell, R Davies, G Batterby. Department of Neurology, Royal Preston Hospital, Preston and Centre for Applied Statistics, and Department of Geography, University of Lancaster, UK

The results of epidemiological studies in motor neuron disease (MND) to date have been confused by wildly dichotomous conclusions. A prospective case control study of MND in Lancashire and South Cumbria over a five year period has been undertaken primarily aimed at determining whether MND shows geographical aggregation. This was supplemented by a detailed life profile questionnaire and the results of a preliminary risk factor analysis for 128 patients with MND are described here.

Onset tended to be earlier in males and as age of onset increased, survival shortened. The main findings, however, centred on childhood viral infections. Patients with MND were less likely to report previous chicken pox (odds ratio $0.53,95 \%$ confidence interval ( $95 \%$ CI) $0.30-0.94)$ than healthy age and sex matched controls and patients with MND who had had chicken pox tended to survive longer after diagnosis than those who did not recall this disease. Furthermore, patients with MND were less likely to be only children (odds ratio 0.33 , 95\% CI 0.15-0.72) and were also less likely to be the first born in a multiple sibship (odds ratio $0.48,95 \%$ CI $0 \cdot 27-0.77$ ). The explanation of these unexpected findings is uncertain, but they may relate to exposure to viruses during early life.

IN VIVO STUDIES OF MOTOR NEURONAL METABOLISM IN MOTOR NEURON DISEASE USING PROTON MAGNETIC RESONANCE SPECTROSCOPY

JD Mitchell, AP Jones, WJ Gunawardena, CMA Coutinho, JA Gatt, J Knight, IC 
Shaw. Departments of Neurology, Neuroradiology, Clinical Chemistry and Medical Physics, Royal Preston Hospital, Preston, and Centre for Toxicology, University of Central Lancashire, Preston, UK

Proton $\left({ }^{1} \mathrm{H}\right)$ magnetic resonance spectroscopy (MRS) offers unparalleled opportunities for the non-invasive study of human metabolism in vivo. Following work on ${ }^{32} \mathrm{P}$ MRS in metabolic muscle disease attention has now moved to ${ }^{1} \mathrm{H}$ MRS. It is possible to study substances such as $\mathrm{N}$-acetylaspartate (NAA), choline (Cho) and creatine (Cr) in the human motor cortex in vivo with a 20 $\mathrm{mm}^{3}$ voxel using ${ }^{1} \mathrm{H}$ MRS.

Seven patients with $M N D$ and seven healthy controls were examined by ${ }^{~} H$ MRS using the Siemens 1.5 Tesla MR Scanner at the Royal Preston Hospital. NAA/Cr $(\mathbf{P}=$ $0.025)$ and NAA/Cho $(P=0.04)$ ratios were reduced in the patients with $\mathrm{MND}$. Attempts have been made to examine the cervical cord using an even smaller voxel. This methodology has been further developed to encompass Stimulated Echo (STEAM) technology in an attempt to study an even wider range of substances such as lipids. It is hoped that this programme will result in validated methods for the in vivo study of excitotoxicity and free radical mechanisms in $M N D$, and that further insights into pathogenetic mechanisms in this devastating disease will be obtained from this work. 